\title{
The burden of miscarriages and perinatal deaths in Sierra Leone, data from a nation-wide household survey (PRESSCO 2020)
}

\author{
Hanna Matheron ${ }^{1}$, Josien Westendorp ${ }^{2}$, Alex van Duinen ${ }^{3}$, Momoh Mansaray $^{4}$, Lora \\ Daskalska $^{5}$, Osman Sankoh ${ }^{6}$, Håkon Bolkan ${ }^{3}$, Martin Grobusch $^{7}$, and Reinou Groen ${ }^{8}$ \\ ${ }^{1}$ University of Amsterdam \\ ${ }^{2}$ Academic Medical Center of Utrecht \\ ${ }^{3}$ Norwegian University of Science and Technology \\ ${ }^{4}$ Masanga Medical Research Unit, Masanga, Tonkolili District, Sierra Leone \\ ${ }^{5}$ Institute for Health and Society and Epidemiology Division, Medical College of Wisconsin, \\ Milwaukee, WI, USA \\ ${ }^{6}$ Statistics Sierra Leone, Freetown, Sierra Leone \\ ${ }^{7}$ Center of Tropical Medicine and Travel Medicine, Amsterdam University Medical Centers \\ ${ }^{8}$ Johns Hopkins Hospital
}

January 29, 2022

\begin{abstract}
Objective: This study identified the prevalence of miscarriages and perinatal deaths from a community perspective. Design: A population-based, cross-sectional household study. Setting: Sierra Leone (West-Africa) from 2019-2020 Population: Women of reproductive age Methods: Seventy-five randomized clusters were selected, 25 households in each cluster and two random participants from each household. Female participants between 12 and 50 years of age were interviewed about family planning methods and obstetrical history. Main outcome measures: miscarriage and perinatal death Results: A total of 914 women of reproductive age were included. Family planning methods were used by $29 \%$ of the women. In total, 2,160 life-time pregnancies were recalled of which $104(5 \%, 95 \%$ CI 4-6\%) resulted in an early pregnancy loss. Eighty-three (80\%) were reported as spontaneous abortion, $17 \%$ (18) were intentional abortions, of which 9 were induced with medication outside the hospital. The stillbirth rate was 18.5 (95\% CI 9-31) per 1000 births and the perinatal mortality rate was 38,4 (95\% CI 24-54) per 1000 births calculated over a 5 -year period. Conclusion: This study confirms that the perinatal death rate in Sierra Leone exceeds the global average. The miscarriages both spontaneous and intentional, are likely to be underreported. Medical attention should be placed to treat and investigate the causes of miscarriages and perinatal deaths. Improving the use of family planning is needed to decrease the hidden burden of intentional abortions. Funding: Norwegian University of Science and Technology (NTNU) and University of Amsterdam's Center of Tropical Medicine and Travel Medicine.
\end{abstract}

\section{Hosted file}

PRESSCO 2020 Miscarriages and Perinatal deaths in Sierra Leone.docx available at https: //authorea. com/users/457980/articles/554598-the-burden-of-miscarriages-and-perinataldeaths-in-sierra-leone-data-from-a-nation-wide-household-survey-pressco-2020 